\title{
The perception of empty and filled time intervals by pigeons
}

\author{
Andrew Miki and Angelo Santi \\ Wilfrid Laurier University, Waterloo, Canada
}

\begin{abstract}
Pigeons were trained in a within-subjects design to discriminate durations of a filled interval ( $2 \mathrm{~s}$ and $8 \mathrm{~s}$ of light) and durations of an empty interval ( $2 \mathrm{~s}$ and $8 \mathrm{~s}$ bound by two $500-\mathrm{ms}$ light markers). Filled intervals required a response to one set of comparisons (e.g., blue vs. yellow), whereas empty intervals required a response to a different set of comparisons (e.g., red vs. green). Psychophysical testing indicated that empty intervals were judged to be longer than equivalent durations of a filled interval. This finding was replicated when the anchor durations used during training were changed to $1 \mathrm{~s}$ and $4 \mathrm{~s}$, or $4 \mathrm{~s}$ and $16 \mathrm{~s}$. The difference between the point of subjective equality (PSE) for the empty intervals and the PSE for filled intervals increased as the magnitude of the anchor duration pairs increased. In addition, the difference limens (DL) for empty intervals were smaller than those for filled intervals, and they also increased as the magnitude of anchor duration pairs increased. An analysis of the Weber fractions (WF; i.e., DL/PSE) provided evidence for superimposition of the empty and filled timing functions across the different sets of anchor durations. These results suggest that the accumulation of subjective time was greater for empty intervals than for filled intervals. Within the framework of scalar timing theory, this difference in timing appeared to be the result of a clock rate difference rather than a switch latency difference.
\end{abstract}

The ability of humans and animals to perceive and remember time has often been investigated with a temporal bisection procedure. In this procedure, subjects are trained to choose between two responses ("short" or "long") following one of two training durations. Once a high level of accuracy is achieved, intermediate durations are introduced, but responses on these trials are not reinforced. From this procedure, a psychophysical function can be generated, and the point of subjective equality (PSE) can be calculated. The PSE is the value on the time dimension at which the subject displays indifference between choosing the "short" or the "long" response. Although studies with animals (Church \& Deluty, 1977; Gibbon, 1986)

Correspondence should be addressed to A. Santi, Department of Psychology, Wilfrid Laurier University, Waterloo, Ontario, Canada, N2L 3C5. Email: asanti@wlu.ca

This research was supported by Grant OGPOOD6378 from the Natural Sciences and Engineering Research Council of Canada to A. S. The authors thank Marion Corrick for her technical assistance. These are based on a portion of a master's thesis submitted by A. Miki to Wilfrid Laurier University. Andrew Miki is currently in the Department of Psychology at York University, Toronto, Ontario.

(C) 2005 The Experimental Psychology Society

http://www.tandf.co.uk/journals/pp/02724995.html

DOI:10.1080/02724990444000032 
have frequently reported that the PSE is at the geometric mean of the two training durations, studies with humans have found the PSE to be either at the arithmetic mean (Wearden, 1991; Wearden \& Ferrara, 1995, 1996; Wearden, Rogers, \& Thomas, 1997) or at the geometric mean (Allan, 1992; Allan \& Gibbon, 1991).

Nontemporal signal properties have been shown to play an important role in human time perception (Allan, 1979; Goldstone \& Lhamon, 1974; Grondin, 1993; Poynter, 1989). For example, auditory signals are judged to be longer in duration than visual signals when humans time both an auditory and a visual signal in the same session (Penney, Gibbon, \& Meck, 2000; Wearden, Edwards, Fakhri, \& Percival, 1998). Animals' temporal judgements are also affected by nontemporal stimulus factors (Buhusi \& Meck, 2000; Buhusi, Sasaki, \& Meck, 2002; Fetterman, 1996; Stubbs, Dreyfus, \& Fetterman, 1984). Temporal discrimination accuracy in animals is affected by stimulus modality (Meck, 1984; Roberts, Cheng, \& Cohen, 1989; Stubbs et al., 1984), stimulus intensity (Kraemer, Brown, \& Randall, 1995; Wilkie, 1987), and duration of food access versus light duration (Spetch \& Wilkie, 1982). Pigeons time visual signals more accurately than auditory signals (Roberts et al., 1989), whereas the reverse is found for rats (Meck, 1984). Rats also appear to judge auditory signals as being longer than visual signals of the same duration (Meck, 1991).

Whether the interval to be timed is filled or empty also affects temporal perception in both humans (Abel, 1972a, 1972b; Grondin, 1993; Rammsayer \& Lima, 1991) and pigeons (Mantanus, 1981). Although Mantanus (1981) reported that pigeons' temporal discriminations were more accurate with filled intervals than with empty intervals, the interpretation of this effect is ambiguous because of a number of design and general test procedure problems, which were described by Kraemer, Randall, and Brown (1997). In order to address these concerns, Kraemer et al. (1997) conducted a study in which pigeons were trained to discriminate either filled intervals (light present) or empty intervals (light absent). They found that pigeons judged the duration of a filled interval to be longer than that of an empty interval of equivalent physical duration. This result is consistent with the finding of Mantanus (1981) because, at the intermediate and at the longest stimulus durations, the filled interval resulted in a higher percentage of long responses than did the empty interval. Thus, accuracy at the long stimulus duration was higher for filled intervals than for empty intervals. However, the Kraemer et al. finding is surprising in that it was based on a between-subjects comparison. Penney et al. (2000) have argued that the internal clock model of timing assumes that the internal clock runs at different rates for stimuli with different properties, and that the detection of a timing difference requires that the accumulator values for the different signal properties be stored within a single reference memory distribution representing the short values for the two signal properties and within a single reference memory distribution representing long values for the two signal properties. This mixing of signal properties within the short and the long reference memory distributions would presumably only be possible if the two different types of signal were timed within the same session. Although the signal properties that they specifically considered involved different stimulus modalities (auditory and visual), similar theoretical mechanisms would be expected to underlie timing differences based on other nontemporal signal characteristics, such as whether the interval is filled or empty. Consistent with theoretical expectations of the mixed memory model, Penney et al. (2000) reported that differences in the timing of auditory and visual signals in humans was obtained when the same subjects experienced the different stimulus conditions 
within the same session (within-subject design), but not when different subjects were tested under the different stimulus conditions (between-subject design). While Penney et al. (2000) acknowledged that some between-subject designs have found a modality difference, they pointed out that in these studies participants were required to compare auditory and visual signals to the same previously established standard (e.g., a clock second). Comparing signals relative to the same previously established standard means that signals timed with a fast clock will seem relatively longer than the standard, and, conversely, signals timed with a slow clock will seem relatively slower than the standard. However, when signals timed with a fast clock are compared to memory distributions for a signal timed with an equally fast clock, and signals timed with a slower clock are compared to memory distributions for a signal timed with an equally slow clock, no classification differences would be expected. Consequently, from the perspective of the internal clock model with mixed memories (Penney et al., 2000), it is surprising that Kraemer et al.'s (1997) between-subject methodology produced a timing difference between filled and empty intervals in pigeons.

The present experiment was conducted to assess the perception of filled and empty time intervals in pigeons using a within-subject design. Pigeons were trained to discriminate durations of a filled interval ( 2 and $8 \mathrm{~s}$ of light), and durations of an empty interval ( $2 \mathrm{~s}$ and $8 \mathrm{~s}$ bound by two $500-\mathrm{ms}$ light markers). Filled intervals required a response to one set of comparisons (e.g., blue vs. yellow), whereas empty intervals required a response to a different set of comparisons (e.g., red vs. green). During psychophysical testing, intermediate durations $(2.6,3.2,4.0,5.0$, and $6.4 \mathrm{~s})$ were presented along with the anchor durations. The pigeons were then retrained with two additional pairs of anchor durations ( 1 and $4 \mathrm{~s}$ or 4 and $16 \mathrm{~s}$ ), and additional psychophysical testing was conducted. Training with different sets of anchor durations helps to differentiate a switch onset latency effect from a clock rate effect (Penney et al., 2000; Wearden et al., 1998). Switch onset latency effects are independent of the anchor durations employed and additive. Hence, the switch onset latency account anticipates that any difference between the PSE for filled intervals and the PSE for empty intervals would remain constant across the different sets of anchor durations. Conversely, clock rate effects are multiplicative with real time. Thus, the clock rate difference hypothesis predicts that the difference between the PSE for filled intervals and the PSE for empty intervals would increase as the anchor duration values increased.

\section{Method}

\section{Subjects}

Nine White Carneaux pigeons, maintained at approximately $80 \%$ of their ad lib weights and housed individually with constant access to grit and water, served as subjects. The colony room was illuminated on a 12:12-hr light:dark cycle by fluorescent lights. The subjects were tested five times a week during the light cycle. All of the birds had prior experience with delayed matching-to-sample involving colour and/or line stimuli in operant chambers, but none had served in experiments involving the timing of visual stimuli in a touchscreen chamber.

\section{Apparatus}

Three touchscreen testing stations located in individual test rooms were used. Each test station consisted of a clear Plexiglas cage $(30 \mathrm{~cm}$ wide $\times 40 \mathrm{~cm}$ deep $\times 36 \mathrm{~cm}$ high $)$ with a large opening cut 
into the one end wall that was constructed of stainless steel. On both the left and the right side walls adjacent to the stainless steel wall, there were openings of $5.7 \times 5 \mathrm{~cm}$ that provided access to a hopper filled with mixed grain (Coulbourn Model E14-10). A colour SuperVGA monitor (Mitsubishi SD4311C) with an attached touch frame (Carrol Touch Inc., Frame 8100-9583-01, Card 8200-3224-01) was placed against the opening of the stainless steel wall. An IBM-compatible microcomputer, located in the same room, controlled stimulus displays, recorded peck locations, and operated feeders.

\section{Procedure}

Pretraining. All birds were trained to eat mixed grain from both the right and the left illuminated food hoppers and to peck at stimuli presented on the monitor. During these preliminary training sessions, a homogeneous grey square, $3.4 \times 3.2 \mathrm{~cm}$ (width $\times$ height), was presented on either the left or the right side of the monitor. A response to the grey square or an elapsed time interval without a response provided access to mixed grain randomly presented at either the left or the right hopper with a probability of 0.5 . The experimenter manipulated both the presentation duration of the grey square and the duration of access to mixed grain during pretraining. This pretraining continued until the pigeons were reliably pecking at both the left and right response areas and eating with only 4-s access to the hopper.

Training with 2- and 8-s anchor durations. After pretraining, the birds were trained to discriminate between short (2-s) and long (8-s) durations of filled and empty intervals of light. The visual stimulus consisted of the presentation of a homogeneous grey square, $3.3 \times 3.3 \mathrm{~cm}$, in the central area of the monitor (approximately $12 \mathrm{~cm}$ from the left and right bezel, as measured to the nearest edge). On filled interval trials, the grey square was presented for either 2 or $8 \mathrm{~s}$. On empty interval trials, the grey square was presented for $500 \mathrm{~ms}$ at the beginning and at the end of a 2- or 8-s unfilled interval. Comparison stimuli were presented in two rectangular response areas, each measuring approximately $3.4 \mathrm{~cm} \times 3.2 \mathrm{~cm}$ (width $\times$ height), one on the left and one on the right side of the monitor (approximately $15.6 \mathrm{~cm}$ apart, as measured from their inside edges). Position of the colour comparison stimuli was counterbalanced over trials. For five of the birds, red and green comparisons were presented after empty intervals, and blue and yellow comparisons were presented after filled intervals. For four birds, blue and yellow comparisons were presented after empty intervals, and red and green comparisons were presented after filled intervals. The comparison stimulus that was designated correct following the short and the long duration signal was also balanced across birds. One of the eight different combinations of comparison stimuli designated as correct following the short and long signals was randomly assigned to each bird. For example, one of the eight combinations was as follows: Green was correct following the short empty interval, red was correct following the long empty interval, yellow was correct following the short filled interval, and blue was correct following the long filled interval. Because there were nine birds, two of the birds received the same combination. The relationship between the type of interval (filled or empty), duration of the interval (short or long), and corresponding correct comparison stimulus (red, green, blue, and yellow) remained constant for each bird throughout this experiment.

For all the birds, a single response to one of the comparison stimuli turned them off and, if correct, provided 4-s access to mixed grain randomly presented at either the left or the right hopper opening with a probability of .5. Incorrect responses to the comparison stimuli resulted in a 4-s blackout, followed immediately by re-presentation of the same interval duration and comparison stimulus configuration. A correct response on a correction trial resulted in 4-s access to mixed grain, although only the choice response on the initial (noncorrection) trial was used to calculate matching accuracy. When necessary, supplementary feedings of Purina Pigeon Chow occurred after the experimental sessions 
and on days when the birds were not run. Within each block of eight trials, all combinations of the four sample stimuli (two interval types by two signal durations) and the two comparison stimulus configurations occurred once. The order of presentation was randomized individually for each bird. Training continued for 40 sessions (except for one bird that only received 35 sessions) with 160 trials per session. The duration of the intertrial interval (ITI) randomly varied within sessions $(4,8,16$, or $32 \mathrm{~s})$. No illumination was presented in the testing chamber during the ITI. At the end of training, eight birds were able to discriminate between the stimuli with at least $85 \%$ accuracy, while one bird achieved $78 \%$ accuracy.

Psychophysical testing with 2- and 8-s anchor durations. Each psychophysical test session consisted of 160 trials. The anchor durations were presented on 80 trials and intermediate durations on 80 trials. The intermediate durations were 2.6, 3.2, 4.0, 5.0, and $6.4 \mathrm{~s}$. In each session, each intermediate duration was randomly tested 8 times for each type of interval (filled or empty). The pigeons were still reinforced for responding correctly following the four training signals (two interval types by two signal durations), but responses following intermediate signals were never reinforced. All other aspects of these sessions were the same as those described above.

Training and psychophysical testing with 1- and 4-s or 4- and 16-s anchor durations. After the psychophysical testing described above, the birds were retrained (eight for 15 sessions and one for 24 sessions) with $1-$ and 4-s anchor durations. The filled and empty intervals were similar to those previously described except for the change in duration. All other training procedures were similar to those described earlier. The birds were given 15 sessions of psychophysical testing with intermediate durations of $1.3,1.6,2.0,2.5$, and $3.2 \mathrm{~s}$. All aspects of these sessions, except for the duration change, were the same as those previously described.

The birds were then retrained (eight for 36 sessions and one for 22 sessions) with 4 - and 16-s anchor durations and were subsequently given 15 sessions of psychophysical testing with intermediate durations of $3.9,4.8,6.0,7.5$, and $9.6 \mathrm{~s}$. All other test procedures were similar to those previously described.

In all the statistical analyses reported in this article, the rejection region was $p<.05$.

\section{Results}

\section{Acquisition}

The mean percentage of correct responding for filled and empty intervals during acquisition of the initial discrimination between the $2-$ and 8 -s anchor durations is presented in Figure 1. Early in training, accuracy for filled intervals was somewhat greater than accuracy for empty intervals. An analysis of variance (ANOVA) conducted on these data indicated a significant main effect for blocks of sessions, $F(7,56)=87.26$, and a significant interaction of interval type by session blocks, $F(7,56)=3.21$. Simple main effects analysis indicated that accuracy for filled intervals was greater than that for empty intervals only during the second block of sessions, $F(1,8)=6.41$.

Additional analyses were conducted to examine performance during the last five sessions of training with each of the three sets of anchor durations. However, these data are not being presented because at the end of each training phase there were no significant differences as a function of interval type or signal duration, and no significant interactions. Pigeons exhibited equivalent levels of accuracy in timing empty and filled intervals at the anchor durations. 


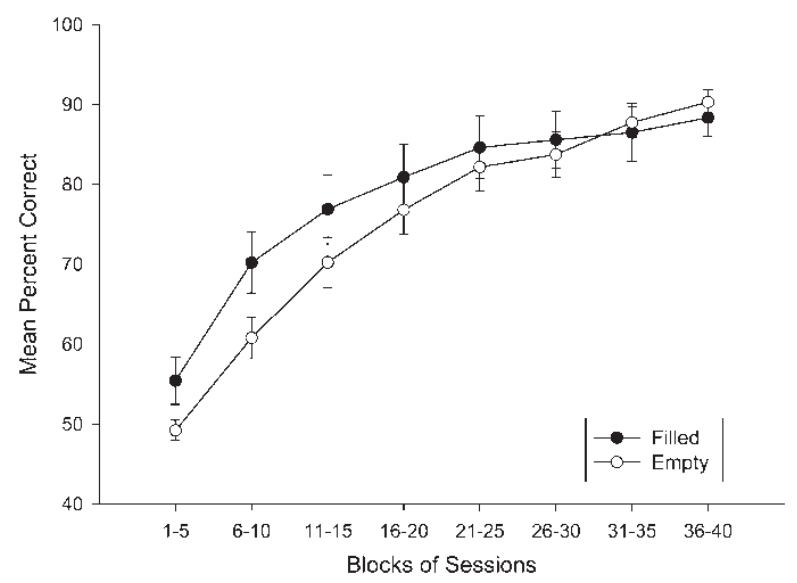

Figure 1. The mean percentage of correct responding for filled and empty intervals during acquisition of the initial discrimination between the 2- and 8-s anchor durations. Error bars represent the standard error of the mean.

\section{Psychophysical testing}

Figure 2 presents the psychophysical functions that were obtained following 1- versus 4-s training (top panel), 2- versus 8-s training (middle panel), and 4- versus 16-s training (bottom panel). The psychophysical function for empty intervals was horizontally displaced to the left of the filled interval function following training with each of the three sets of anchor duration. Empty intervals were perceived as longer than filled intervals of the same duration. An ANOVA of the data in Figure 2 was conducted with anchor duration set, type of interval, and signal duration as within-subject factors. Significant main effects for anchor duration set, $F(2,16)=11.56$, interval type, $F(1,8)=14.36$, and signal duration, $F(6,48)=$ 346.68 , were obtained. The Interval Type $\times$ Signal Duration interaction and the Anchor Duration Set $\times$ Signal Duration interaction were also significant, $F(6,48)=4.87$, and $F(12$, $96)=5.78$, respectively. The three-way interaction of Anchor Duration Set $\times$ Interval Type $\times$ Signal Duration was not statistically significant, $F<1$. Simple main effects analysis of the Interval Type $\times$ Signal Duration interaction did not find any differences between filled and empty intervals at the two shortest or at the two longest signal durations, all $F_{\mathrm{s}}<1.94$. However, the probability of classifying a duration as long was significantly greater for empty intervals than for filled intervals at the three intermediate signal durations in the middle of the functions, $F_{\mathrm{s}}(1,8)=12.15,16.91$, and 9.91. The Anchor Duration Set $\times$ Sample Duration interaction primarily reflected differences in the probability of a long response at specific signal durations across anchor durations. At the shortest signal duration and at the second longest signal duration, there was no difference in the probability of a long response across the different sets of anchor durations. However, at all other signal durations, there were significant differences in the probability of long responding across the anchor duration sets, $F_{\mathrm{s}}(2,16)=6.29,9.57,7.98,19.93$, and 3.74, respectively. In general, this was due to a lower level of long responding for the 1-and 4-s anchor durations than for the two longer anchor duration sets. 


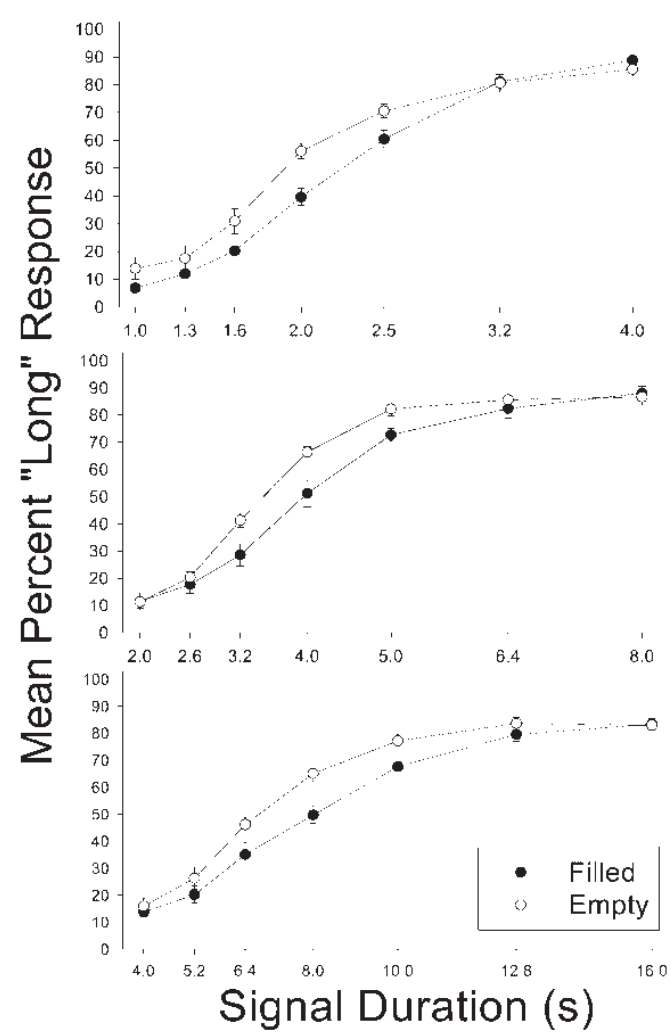

Figure 2. The mean percentage of long responses as a function of signal duration for filled and empty intervals following training with anchor durations of 1 and $4 \mathrm{~s}$ (top panel), 2 and $8 \mathrm{~s}$ (middle panel), and 4 and $16 \mathrm{~s}$ (bottom panel). Error bars represent the standard error of the mean.

The PSE was estimated from the psychophysical functions for each pigeon by conducting linear regressions of the proportion of long responses for each of three adjacent signal durations. The regression equation with the greatest slope for each pigeon was used to estimate the PSE by calculating the signal duration associated with $50 \%$ of the long responses. This method originally employed by Maricq, Roberts, and Church (1981) has been frequently used for animal (e.g., Meck 1986) and human timing data (e.g., Droit-Volet \& Wearden, 2002; Wearden \& Ferrara, 1996; Wearden et al., 1997), and it produces results that are very similar to those obtained with other methods (Wearden \& Ferrara, 1995). The mean PSE for empty and filled intervals for each anchor duration pair is shown in Table 1. The PSEs increased as the magnitude of the anchor duration pair increased and were smaller for empty intervals than for filled intervals. An ANOVA with interval type and anchor duration set as within-subjects factors was conducted on the PSE data. The analysis revealed a significant effect of interval type, $F(1,8)=12.23$, anchor duration set, $F(2,16)=505.91$, and an Interval Type $\times$ Anchor Duration Set interaction, $F(2,16)=3.95$. A simple main effects analysis indicated that the PSE for empty intervals was significantly less than the PSE for 
TABLE 1

Mean PSEs, DLs, and WFs for the empty and filled intervals at each signal duration

\begin{tabular}{|c|c|c|c|c|c|c|c|}
\hline \multirow[b]{2}{*}{ Signal duration ${ }^{\mathrm{a}}$} & \multirow[b]{2}{*}{ Interval type } & \multicolumn{2}{|c|}{$P S E^{\mathrm{a}}$} & \multicolumn{2}{|c|}{$D L$} & \multicolumn{2}{|c|}{$W F$} \\
\hline & & $M$ & $S E M$ & $M$ & $S E M$ & $M$ & $S E M$ \\
\hline \multirow[t]{2}{*}{$1-4$} & Empty & 1.89 & 0.09 & 0.48 & 0.06 & 0.26 & 0.05 \\
\hline & Filled & 2.26 & 0.07 & 0.56 & 0.04 & 0.25 & 0.01 \\
\hline \multirow[t]{2}{*}{$2-8$} & Empty & 3.47 & 0.07 & 0.76 & 0.05 & 0.22 & 0.01 \\
\hline & Filled & 3.99 & 0.15 & 0.94 & 0.09 & 0.24 & 0.02 \\
\hline \multirow[t]{2}{*}{$4-16$} & Empty & 6.64 & 0.22 & 1.62 & 0.16 & 0.26 & 0.03 \\
\hline & Filled & 7.85 & 0.35 & 2.13 & 0.2 & 0.27 & 0.02 \\
\hline
\end{tabular}

Note: $\quad$ PSE $=$ point of subjective equality. $\mathrm{DL}=$ difference limens. WF $=$ Weber fractions.

${ }^{\mathrm{a}}$ In $\mathrm{s}$.

filled intervals for each set of anchor durations, $F_{\mathrm{S}}(1,8)=19.64,17.75$, and 7.32. The significant interaction reflects the increasing difference between the mean PSE for empty intervals and the mean PSE for filled intervals as the magnitude of the anchor duration pair increased.

For the 1- and 4-s anchor durations, the PSE for empty intervals did not differ from the geometric mean of $2.0 \mathrm{~s}, t<1$, but the PSE for filled intervals was significantly greater than the geometric mean, $t(8)=3.81$. For the 2 - and 8 -s anchor durations, the PSE for empty intervals was significantly below the geometric mean of $4.0 \mathrm{~s}, t(8)=-8.16$, while the PSE for filled intervals did not differ from the geometric mean, $t<1$. For the 4 - and 16 -s anchor durations, the PSE for empty intervals was again significantly below the geometric mean of $8.0 \mathrm{~s}, t(8)=-6.29$, and the PSE for filled intervals did not differ from the geometric mean, $t<1$. In general, the PSE for empty intervals was at or below the geometric mean, while the PSE for filled intervals was at or above the geometric mean.

The regression equations were also used to calculate difference limens (DL), which represent the average difference between the signal duration associated with $75 \%$ long responses and the signal duration associated with $25 \%$ long responses. The mean DL for empty and filled intervals at each anchor duration pair is shown in Table 1. The DLs increased as the magnitude of the anchor duration pair increased and tended to be smaller for empty intervals than for filled intervals. An ANOVA of the DL data indicated a significant effect of interval type, $F(1,8)=6.84$, and anchor duration set, $F(2,16)=57.77$. However, the Interval Type $\times$ Anchor Duration Set interaction was not statistically significant. These data indicate that pigeons were more precise in timing empty intervals than in timing filled intervals.

The PSE for each averaged response function was calculated, and the functions were normalized by dividing the signal durations by the PSE. As shown in Figure 3, when the functions were plotted in this relative fashion, the six response functions exhibited reasonable superimposition. This suggests that the difference between the empty and the filled response functions is proportional rather than absolute.

The Weber fraction (WF) was calculated by dividing the DL by the PSE. The WF is a coefficient of variation which provides a measure of timing variability that takes into account 


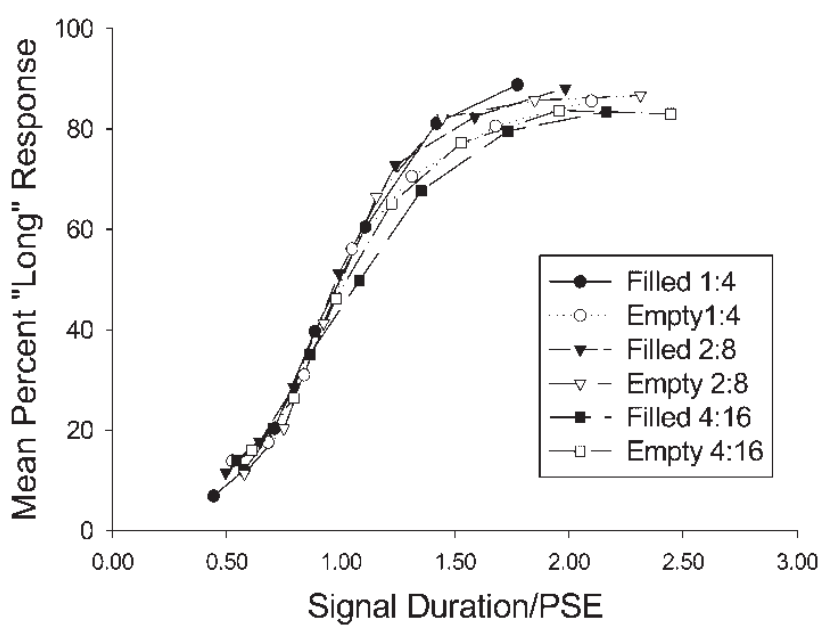

Figure 3. Superimposition plot of averaged response functions for empty and filled intervals. The labels 1:4, 2:8, 4:16 refer to the anchor durations used during training. The values on the $x$-axis represent the signal duration divided by the point of subjective equality.

the duration being timed. According to scalar timing theory, the value of the WF should remain constant across different sets of anchor durations even though the DL increases as the geometric mean of the anchor durations increases. Analysis of the WF values provides a statistical test of the superimposition of timing functions. The mean WF for empty and filled intervals at each anchor duration pair is shown in Table 1 . The WF values were very similar for empty intervals and for filled intervals and also very similar across sets of anchor durations. An ANOVA of the WF data indicated no significant main effects or interaction, $F_{\mathrm{S}}<1.33$. These data provide evidence for superimposition of timing functions across the different sets of anchor durations.

To determine whether the timing difference between empty intervals and filled intervals was due to a difference in the switch latency (slower for filled intervals), or due to a clock rate difference (faster for empty intervals), an analysis of the PSE difference scores was undertaken. If the difference in the PSE between empty and filled intervals remained constant across different anchor durations, a difference in switch latency would be implicated. Conversely, a multiplicative difference in the PSE between empty and filled intervals across training durations would indicate that the timing difference was due to a clock rate difference. For anchor durations of 1 and $4 \mathrm{~s}$, the difference between the PSE for empty intervals $(1.89 \mathrm{~s})$ and filled intervals $(2.26 \mathrm{~s})$ was $0.36 \mathrm{~s}$. If this difference was due to a clock rate difference, rather than a switch latency difference, the PSE difference score between empty and filled intervals following training with anchor durations of 2 and $8 \mathrm{~s}$ should be twice this value (i.e., approximately $0.72 \mathrm{~s}$ ). Similarly, the PSE difference score following training with anchor durations of 4 and $16 \mathrm{~s}$ should be double the difference obtained following training with anchor durations of 2 and $8 \mathrm{~s}$ (i.e., $1.44 \mathrm{~s}$ ). On the other hand, if the timing difference was due to a switch latency difference, then the PSE difference score should remain the same regardless of the anchor durations used in training. 
An ANOVA was conducted to compare the PSE difference scores across the three sets of anchor durations. A significant difference was found, $F(2,16)=3.95$, and the means were compared by use of a protected $t$-statistic. Although the mean PSE difference following training with 1 - and 4-s anchors $(M=0.36 \mathrm{~s}, S E M=0.08)$ was less than the mean PSE difference following training with 2 - and $8-\mathrm{s}$ anchors $(M=0.52 \mathrm{~s}, S E M=0.12)$, the difference was not statistically significant. However, the mean PSE difference following training with 4- and 16-s anchors $(M=1.21 \mathrm{~s}, S E M=0.45)$ was significantly greater than the mean PSE difference following training with either the 2- and 8-s anchors, or the 1- and 4-s anchors, $t \mathrm{~s}(16)=2.14,2.65$. The PSE difference following training with the $4-$ and $16-\mathrm{s}$ anchors was more than double that obtained for the 2 - and 8 -s anchors and more than triple the value for the 1- and 4-s anchors. These data are inconsistent with a switch-latency effect, because the PSE difference score should have remained the same regardless of the anchor durations used in training. However, they do provide some support for a clock rate effect. The rankordering of the PSE difference for the different sets of anchor durations was consistent with a clock rate effect, and the PSE difference for the 4- and 16-s anchor pair was significantly greater than the PSE difference for either the 2-and 8-s pair or the 1- and 4-s pair. The only problem for the clock rate hypothesis is that the mean PSE difference following training with 1- and 4-s anchors was not significantly less than the mean PSE difference following training with 2 - and 8 -s anchors. However, the observed difference was in the direction predicted by the clock rate hypothesis.

\section{DISCUSSION}

The present study assessed the perception of filled and empty time intervals in pigeons using a within-subjects design. The psychophysical functions and the PSE measures derived from them indicated that the empty interval bisection function was displaced horizontally to the left of the filled interval function. This suggests that the accumulation of subjective time was greater for empty intervals than for filled intervals. Although the direction of the difference is opposite from that reported in previous pigeon studies (Kraemer et al., 1997; Mantanus, 1981), the methodology of the current study was stronger in several respects. Unlike the previous studies, the current study operationalized an empty interval in the same way as has the human research (Grondin, Ivry, Franz, Perreault, \& Metthe, 1996), a withinsubject design rather than a between-subject design was used (Penney et al., 2000), and psychophysical testing was conducted for different sets of anchor durations.

The finding that pigeons judge an empty interval to be longer than a filled interval of the same duration is surprising given that humans often judge filled intervals to be longer than empty intervals (Rammsayer \& Lima, 1991; Thomas \& Brown, 1974). However, the difference between pigeons and humans may reflect procedural variables. Grondin (1993) has noted that, in humans, differences in the discrimination of duration for filled and empty intervals depends on the type of markers, the range of durations studied, and the timing task. In some cases, humans discriminate empty intervals more accurately than filled intervals.

The difference in perceived duration of empty and filled intervals did not appear to be due to a switch-latency effect, because the difference between the PSE for empty and for filled intervals did not remain constant across the different pairs of anchor durations. The results are more consistent with a clock rate difference for empty and filled intervals. 
Additional support for this conclusion is provided by the DL data. If the accumulation of subjective time units is greater for empty intervals than for filled intervals, then temporal resolution should be higher for empty intervals than for filled intervals. As a result, the DLs for empty intervals should be smaller than those for filled intervals. A switch latency onset difference would not result in different DLs for empty and filled intervals. In the present study, the mean DL for empty intervals was smaller than those for filled intervals across all anchor duration sets. This supports a clock rate effect. It is also important to note that a clock rate effect predicts a proportional shift in the bisection functions for empty and filled intervals. A proportional shift in the functions would result in superimposition. If the shift was constant as predicted by a switch-latency difference, superimposition of the bisection functions would not be obtained. The analysis of the WFs supported the prediction of a proportional shift and superimposition of the empty and filled interval functions.

The clock rate effect observed in this study could be due to a pacemaker rate difference or an attentional difference in maintaining closure of the timing switch (the flickering switch hypothesis). However, this would require a higher pacemaker rate or less switch flicker for empty than for filled intervals. Given previous animal research findings, this seems counterintuitive. For example, it has been reported in both pigeons (Wilkie, 1987) and rats (Kraemer et al., 1995) that more intense signals are judged to be longer in duration than less intense signals. More intense signals would presumably drive the pacemaker at a faster rate or capture attention more easily so that the switch would flicker less. Since the physical intensity of a filled interval is greater than that of an empty interval in the present experiment, one might have expected filled intervals to be perceived as longer than empty intervals.

While a definitive explanation of the present findings requires additional research, one possible explanation of the present findings can be suggested. Engaging in a nontemporal information processing while timing has been shown to result in the underestimation of time intervals by humans (Block \& Zakay, 1997; Zakay \& Block, 1996, 1997), and animals (Lejeune, Macar, \& Zakay, 1999). Explanations for this effect have been in terms of attentional resources being diverted away from timing. Sutton and Roberts (2002) noted that, in most timing tasks, an animal is required to divide attention between processing the temporal properties of the stimulus and processing other, nontemporal, features of the stimulus or environment. It may be that empty intervals of darkness allowed the pigeons to focus attention on timing signal duration with no competition from nontemporal (visual) features in the chamber. Filled intervals of light, on the other hand, might allow for visual features in the chamber to attract attention, thereby reducing attention to processing the temporal properties of the signal. During the timing of an illuminated filled interval, the switch gating pulses to the accumulator may oscillate between a closed and an open state as a result of attention switching to visual stimuli in the chamber. Empty intervals preclude the presence of visual features in the chamber and thus may be able to maintain the switch in a closed state more easily than does an illuminated filled interval. This would result in an underestimation of filled intervals relative to empty intervals.

The procedures used in the current study have some features that merit comment. The same stimulus was used to signal filled intervals and to mark empty intervals. Consequently, it is possible that the birds simply began timing from the onset to the offset of this stimulus on both filled and empty intervals. If this were the case, all empty intervals would then be $1 \mathrm{~s}$ longer than filled intervals, and it might be expected that pigeons would make more 
"long" responses on these trials. However, this 1-s difference in duration between empty and filled intervals would remain constant across the different sets of anchor durations, and it should result in a constant PSE difference between empty and filled intervals across the anchor duration sets. The psychophysical data indicated that there was a proportional shift in the PSE across anchor durations and not an absolute shift. This finding is inconsistent with the suggestion that pigeons timed the markers as well as the unfilled interval on empty interval trials. Nevertheless, it would be worthwhile to repeat these experiments with signals and markers that were different for filled and for empty intervals.

Another feature of our study was that the ambient stimulus condition during the ITI was the same as that during the empty intervals. Kaiser, Zentall, and Neiman (2002) have presented data on instructional ambiguity and gap effects in the timing of fixed intervals by pigeons, which is consistent with the assumption that pigeons use the ITI to reset their memory of the event duration from the preceding trial. In our study, the long empty intervals $(8 s)$ rather than the short empty intervals $(2 s)$ would be more like the ITIs, which had a minimum value of $4 \mathrm{~s}$ and a mean value of $15 \mathrm{~s}$. This raises the possibility that pigeons may reset their internal clock during long empty interval trials because of their similarity to the ITI. However, this resetting of time during some of the long empty interval trials would produce an underestimation of time relative to filled intervals of the same duration. Hence, it predicts a result opposite to that found.

While the initial acquisition data suggests that the discrimination of empty intervals was somewhat more difficult than the discrimination of filled intervals, the difference was only present early in acquisition. There was no significant difference in accuracy for filled and empty intervals during Blocks 3-8 of acquisition. It is also important to note that the analysis of the psychophysical functions indicated that there was no difference in the probability of a "long" response between filled and empty intervals at the shortest or at the longest signal durations. This indicates that the pigeons classified the anchor durations as accurately on empty interval trials as on filled interval trials across the different anchor duration sets. Thus there is little evidence in these data that the use of the same stimulus for filled and empty intervals, or the similarity of the ITI and empty intervals, made the discrimination of empty intervals substantially more difficult than the discrimination of filled intervals.

Several interpretative frameworks have been developed to explain the results of animal studies on the perception and memory of time intervals. These include scalar timing theory (Gibbon, Church, \& Meck, 1984), the behavioural theory of timing (Killeen \& Fetterman, 1988; Machado, 1997), spectral timing theory (Grossberg \& Schmajuk, 1989), neural network models (Church \& Broadbent, 1990; Dragoi, Staddon, Palmer, \& Buhusi, 2003), and multiple time scale theory (Staddon \& Higa, 1999). Scalar timing theory has been the most dominant theory of interval timing, which has been used to explain the results of both human and animal timing experiments (Allan, 1992, 1998; Allan \& Gibbon, 1991; Ferrara, Lejeune, \& Wearden, 1997; Penney et al., 2000; Wearden, 1991). The present results were interpreted from the framework of scalar timing theory because a significant portion of prior published research examining the effects of stimulus properties on timing has been presented and discussed primarily within this theoretical context (see Droit-Volet, Tourret, \& Wearden, 2004; Grondin, 1993; Penney et al., 2000; Wearden et al., 1998; Wilkie, 1987). It is likely that one or more of the alternative models of timing could provide an equally plausible interpretation of the current set of findings. Future research in our laboratory on the timing of filled and 
empty intervals, using a variety of techniques, will be undertaken to provide more definitive evidence in favour of the mixed-memory version of scalar timing.

In summary, this article investigated the timing of filled and empty intervals in pigeons. Pigeons judged empty intervals to be longer than equal-length filled intervals. The psychophysical functions, the PSE data, and the DL data indicated that the accumulation of subjective time was greater for empty intervals than for filled intervals. The psychophysical functions for filled and empty intervals exhibited good superimposition, and there was no significant difference in the WF across interval type or sets of anchor durations. Thus, the timing difference between empty and filled intervals appears to be the result of a clock rate effect rather than a switch onset latency difference.

\section{REFERENCES}

Abel, S. M. (1972a). Discrimination of temporal gaps. Fournal of the Acoustical Society of America, 52, 519-524.

Abel, S. M. (1972b). Duration discrimination of noise and tone bursts. Fournal of the Acoustical Society of America, 51, 1219-1223.

Allan, L. G. (1979). The perception of time. Perception and Psychophysics, 26, 340-354.

Allan, L. G. (1992). The internal clock revisited. In F. Macar, V. Pouthas, \& W. Friedman (Eds.), Time, action and cognition: Tomards bridging the gap (pp. 191-202). Dordrecht, Netherlands: Kluwer.

Allan, L. G. (1998). The influence of the scalar timing model on human timing research. Behavioural Processes, 44, $101-117$.

Allan, L. G., \& Gibbon, J. (1991). Human bisection at the geometric mean. Learning and Motivation, 22, 39-58.

Block, R. A., \& Zakay, D. (1997). Prospective and retrospective duration judgements: A meta-analytic review. Psychonomic Bulletin \& Reviem, 4, 184-197.

Buhusi, C. V., \& Meck, W. H. (2000). Timing for the absence of a stimulus: The gap paradigm reversed. Fournal of Experimental Psychology: Animal Behavior Processes, 26, 305-322.

Buhusi, C. V., Sasaki, A., \& Meck, W. H. (2002). Temporal integration as a function of signal and gap intensity in rats (Rattus norvegicus) and pigeons (Columbia livia). Journal of Comparative Psychology, 116, 381-390.

Church, R. M., \& Broadbent, H. A. (1990). Alternative representations of time, number and rate. Cognition, 37, 55-81.

Church, R. M., \& Deluty, M. Z. (1977). Bisection of temporal intervals. Fournal of Experimental Psychology: Animal Behavior Processes, 8, 165-186.

Dragoi, V., Staddon, J. E. R., Palmer, R. G., \& Buhusi, C. V. (2003). Interval timing as an emergent learning property. Psychological Reviem, 110, 126-144.

Droit-Volet, S., Tourret, S., \& Wearden, J. (2004). Perception of the duration of auditory and visual stimuli in children and adults. Quarterly Fournal of Experimental Psychology, 57A, 797-818.

Droit-Volet, S., \& Wearden, J. (2002). Speeding up an internal clock in children? Effects of visual flicker on subjective duration. Quarterly Fournal of Experimental Psychology, 55B, 193-211.

Ferrara, A., Lejeune, H., \& Wearden, J. H. (1997). Changing sensitivity to duration in human scalar timing: An experiment, a review, and some possible explanations. Quarterly Fournal of Experimental Psychology, 50B, $217-237$.

Fetterman, J. G. (1996). Dimensions of stimulus complexity. Fournal of Experimental Psychology: Animal Behavior Processes, 22, 3-18.

Gibbon, J. (1986). The structure of subjective time: How time flies. In G. Bower (Ed.), The psychology of learning and motivation (Vol. 20, pp. 105-135). New York: Academic Press.

Gibbon, J., Church, R. M., \& Meck, W. H. (1984). Scalar timing in memory. In J. Gibbon \& L. G. Allan (Eds.), Timing and time perception (Vol. 423, pp. 52-77). New York: New York Academy of Sciences.

Goldstone, S., \& Lhamon, W. T. (1974). Studies of the auditory-visual differences in human time judgements: I. Sounds are judged longer than lights. Perceptual and Motor Skills, 39, 63-82.

Grondin, S. (1993). Duration discrimination of empty and filled intervals marked by auditory and visual signals. Perception and Psychophysics, 54, 383-394. 


\section{MIKI AND SANTI}

Grondin, S., Ivry, R. B., Franz, E., Perreault, L., \& Metthe, L. (1996). Markers' influence on the duration discrimination of intermodal intervals. Perception E Psychophysics, 58, 424-433.

Grossberg, S., \& Schmajuk, N. (1989). Neural dynamics of adaptive timing and temporal discrimination during associative learning. Neural Netmorks, 2, 79-102.

Kaiser, D. H., Zentall, T. R., \& Neiman, E. (2002). Timing in pigeons: Effects of the similarity between intertrial interval and gap in a timing signal. Journal of Experimental Psychology: Animal Behavior Processes, 28, 416-422.

Killeen, P. R., \& Fetterman, J. G. (1988). A behavioral theory of timing. Psychological Reviem, 95, 274-295.

Kraemer, P. J., Brown, R. W., \& Randall, C. K. (1995). Signal intensity and duration estimation in rats. Behavioural Processes, 44, 265-268.

Kraemer, P. J., Randall, C. K., \& Brown, R. W. (1997). The influence of stimulus attributes on duration matchingto-sample in pigeons. Animal Learning \& Behavior, 25, 148-157.

Lejeune, H., Macar, F., \& Zakay, D. (1999). Attention and timing: Dual task performance in pigeons. Behavioural Processes, 45, 141-157.

Machado, A. (1997). Learning the temporal dynamics of behavior. Psychological Reviem, 104, 241-265.

Mantanus, H. (1981). Empty and filled interval discrimination by pigeons. Behaviour Analysis Letters, 1, $217-224$.

Maricq, A. V., Roberts, S., \& Church, R. M. (1981). Methamphetamine and time estimation. Fournal of Experimental Psychology: Animal Behavior Processes, 7, 18-30.

Meck, W. H. (1984). Attentional bias between modalities: Effect on the interval clock, memory, and decision stages used in animal time discrimination. In J. Gibbon \& L. Allan (Eds.), Timing and time perception (Vol. 423, pp. 528-541). New York: New York Academy of Sciences.

Meck, W. H. (1986). Affinity for the dopamine D2 receptor predicts neuroleptic potency in decreasing the speed of an internal clock. Pharmacology, Biochemistry E Behavior, 25, 1185-1189.

Meck, W. H. (1991). Modality-specific circadian rhythmicities influence mechanisms of attention and memory for interval timing. Learning and Motivation, 22, 153-179.

Penney, T. B., Gibbon, J., \& Meck, W. H. (2000). Differential effects of auditory and visual signals on clock speed and temporal memory. Fournal of Experimental Psychology: Human Perception and Performance, 26, 1770-1787.

Poynter, W. D. (1989). Judging the duration of time intervals: A process of remembering segments of experience. In I. Levin \& D. Zakay (Eds.), Time and human cognition: A life-span perspective (pp. 305-321). Amsterdam: Elsevier.

Rammsayer, T. H., \& Lima, S. D. (1991). Duration discrimination of filled and empty auditory intervals: Cognitive and perceptual factors. Perception and Psychophysics, 50, 565-574.

Roberts, W. A., Cheng, K., \& Cohen, J. (1989). Timing light and tone signals in pigeons. Fournal of Experimental Psychology: Animal Behavior Processes, 20, 66-78.

Spetch, M. L., \& Wilkie, D. M. (1982). A systematic bias in pigeons' memory for food and light durations. Behaviour Analysis Letters, 2, 267-274.

Staddon, J. E. R., \& Higa, J. J. (1999). Time and memory: Towards a pacemaker-free theory of interval timing. Fournal of the Experimental Analysis of Behavior, 71, 215-251.

Stubbs, D. A., Dreyfus, L. R., \& Fetterman, J. G. (1984). The perception of temporal events. In J. Gibbon \& L. Allan (Eds.), Timing and time perception (pp. 30-42). New York: New York Academy of Sciences.

Sutton, J. E., \& Roberts, W. A. (2002). The effect of nontemporal information processing on time estimation in pigeons. Learning and Motivation, 33, 124-140.

Thomas, E. A., \& Brown, I. (1974). Time perception and the filled-duration illusion. Perception and Psychophysics, $16,449-458$.

Wearden, J. H. (1991). Do humans possess an internal clock with scalar timing properties? Learning and Motivation, $29,59-83$.

Wearden, J. H., Edwards, H., Fakhri, M., \& Percival, A. (1998). Why "sounds are judged longer than lights": Application of a model of the internal clock in humans. Quarterly fournal of Experimental Psychology, 51B, 97-120.

Wearden, J. H., \& Ferrara, A. (1995). Stimulus spacing effects in temporal bisection by humans. Quarterly Fournal of Experimental Psychology, 48B, 289-310.

Wearden, J. H., \& Ferrara, A. (1996). Stimulus range effects in temporal bisection by humans. Quarterly Fournal of Experimental Psychology, 49B, 24-44.

Wearden, J. H., Rogers, P., \& Thomas, R. (1997). Temporal bisection in humans with longer stimulus durations. Quarterly Journal of Experimental Psychology, 50B, 79-94. 
Wilkie, D. M. (1987). Stimulus intensity affects pigeons' timing behavior: Implications for an internal clock model. Animal Learning E Behavior, 15, 35-39.

Zakay, D., \& Block, R. A. (1996). The role of attention in time estimation processes. In M. A. Pastor \& J. Artieda (Eds.), Time, internal clocks, and movement (pp. 143-164). Amsterdam: Elsevier.

Zakay, D., \& Block, R. A. (1997). Temporal cognition. Current Directions in Psychological Science, 6, 12-16.

Original manuscript received 14 April 2004 Accepted revision received 22 fuly 2004 